\title{
Chest wall tumors: Diagnosis, treatment and reconstruction
}

\author{
GUO-QIANG LIN ${ }^{1}$, YING-QIU LI ${ }^{2}$, LING-JIN HUANG ${ }^{1}$, FAN-YAN LUO ${ }^{1}$, HAI-HE JIANG ${ }^{1}$ and WAN-JUN LUO ${ }^{1}$ \\ ${ }^{1}$ Department of Cardiothoracic Surgery, Xiangya Hospital, Central South University; ${ }^{2}$ Faculty of Basic Medicine, \\ Hunan University of Traditional Chinese Medicine, Changsha, Hunan 410008, P.R. China
}

Received June 28, 2014; Accepted January 12, 2015

DOI: $10.3892 /$ etm.2015.2353

\begin{abstract}
The aim of the present study was to determine a suitable procedure for the treatment of chest wall neoplasms with less potential risk and an increased rate of survival. Fifty patients with suspected chest wall malignancies were analyzed using various preliminary investigation tools. Whole-chest scanning was performed in all the patients. The patients were subsequently subjected to biopsies for further confirmation of the neoplasm. All such patients were then treated with a surgical approach and radiation therapy, with a follow-up period lasting up to six years. The majority of the patients showed improved survival rates relative to conventional therapies. The survival rates of patients suffering from osteosarcoma (78\%) were higher those of patients with rhabdomyosarcoma (73\%) and malignant small round cell tumors $(64 \%)$. The survival and the mortality rates of the patients with synovial sarcoma and fibrosarcoma were the same. This study, which was conducted on a small group of patients, has provided guidance for further studies on tumors of the chest wall, which may, in turn, increase the longevity of affected patients.
\end{abstract}

\section{Introduction}

Cancer, also known as malignant neoplasm, comprises a group of diseases differing widely in their cause and biology. Cancer is considered to be one of the major global threats to human health. The occurrence of chest wall cancer in Asian countries is higher than that in Western European countries (1). Epidemiological data from China shows that $>2.2$ million new cancer cases (1.4 million in males, 0.8 million in females) are diagnosed annually, of which $\sim 1.6$ million cases result in mortality (2). Furthermore, cancer has become the number one cause of mortality in China (3).

Neoplasms of the chest wall comprise a heterogeneous group of lesions that are challenging to both diagnose and

Correspondence to: Mr. Hai-He Jiang, Department of Cardiothoracic Surgery, Xiangya Hospital, Central South University, 87 Xiangya Road, Changsha, Hunan 410008, P.R. China E-mail: jianghaihe1979@gmail.com

Key words: chest wall neoplasm, sarcomas, diagnosis, radiotherapy, chemotherapy treat. These neoplasms account for $<5 \%$ of thoracic malignancies and exhibit a varied pathology, as they can arise from any soft tissue or bony structure around the thoracic cavity. Chest wall neoplasms may be either primary or metastatic, with a malignancy rate of $\sim 50 \%$ (1), and either symptomatic or asymptomatic, with $\sim 20 \%$ found incidentally on chest radiograph (4). Malignant tumors typically involve direct invasion or metastases from adjacent thoracic tumors (1). Malignant rib tumors include multiple myeloma, chondrosarcoma, osteosarcoma and Ewing's sarcoma (4). These tumors characteristically manifest as painful, rapidly growing, large, palpable masses. Osteosarcoma with a less favorable prognosis is more malignant than chondrosarcoma.

The diagnosis of suspected chest wall tumors includes a careful history, proper physical examination and a plain chest $\mathrm{X}$-ray, followed by techniques such as chest radiography, computed tomography (CT), magnetic resonance imaging (MRI) and positron emission tomography (1). Although similar imaging features are characteristic of numerous malignant chest wall tumors, knowledge of the typical radiological manifestations of the malignant tumors frequently enables their differentiation from benign chest wall tumors and may allow a specific diagnosis to be established. The $\mathrm{X}$-ray pattern of specific tumors such as Ewing's sarcoma and osteosarcoma, for example, aids in their diagnosis (5). Despite this, in the majority of cases the radiographic features alone are inadequate for the provision of a complete diagnosis and a histological evaluation is therefore required (4). Furthermore, the selection of one method over the other is usually dependent on the size of the lesion, extent of resection, requirement for reconstruction and associated comorbidities. In general, lesions measuring $<5 \mathrm{~cm}$ undergo excisional biopsy and lesions $>5 \mathrm{~cm}$ may either undergo needle aspiration or incisional biopsy (4). In cases of osteosarcoma, rhabdomyosarcoma (RMS), Ewing's sarcoma and other small-cell sarcomas, chemotherapy is normally suggested as a neoadjuvant therapy, and then continued postoperatively depending upon the response of tumor (6). The combined use of chemotherapy and radiotherapy is contentious but has shown efficacy in adjuvant protocols (2). In earlier studies, it was found that patients succumbed due to these life-threatening tumors (7-9); however, recent advances in diagnostic and treatment techniques have improved survival rates $(4,9)$. The present study investigated the diagnosis and treatment of various chest wall neoplasms with various combinations of chemotherapeutic protocols, surgical resection and radio- 


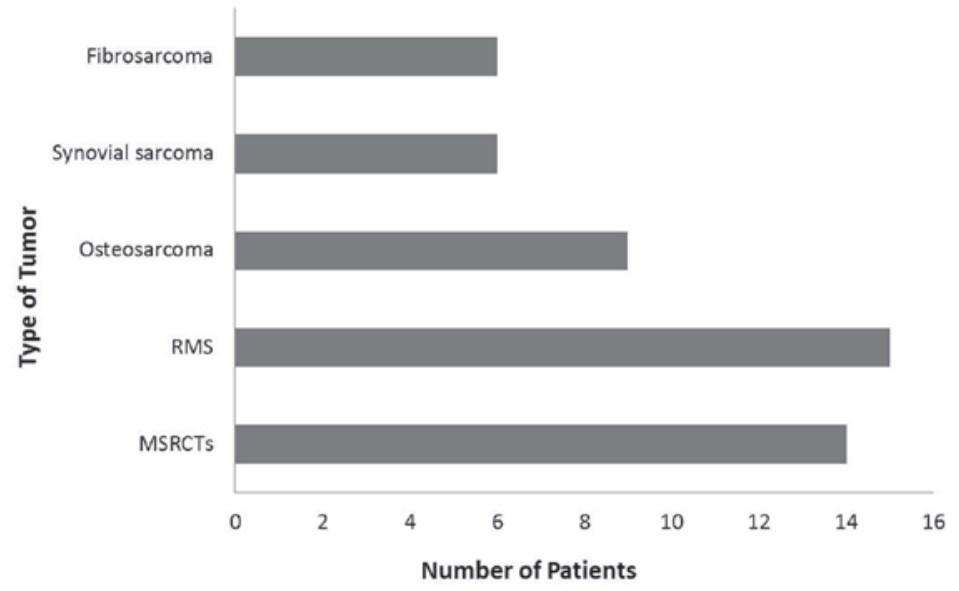

Figure 1. Five groups of patients divided on the basis of tumor type and the confirmation of prognosis following marker-assisted tests. RMS, rhabdomyosarcoma; MSRCT, malignant small round cell tumor.
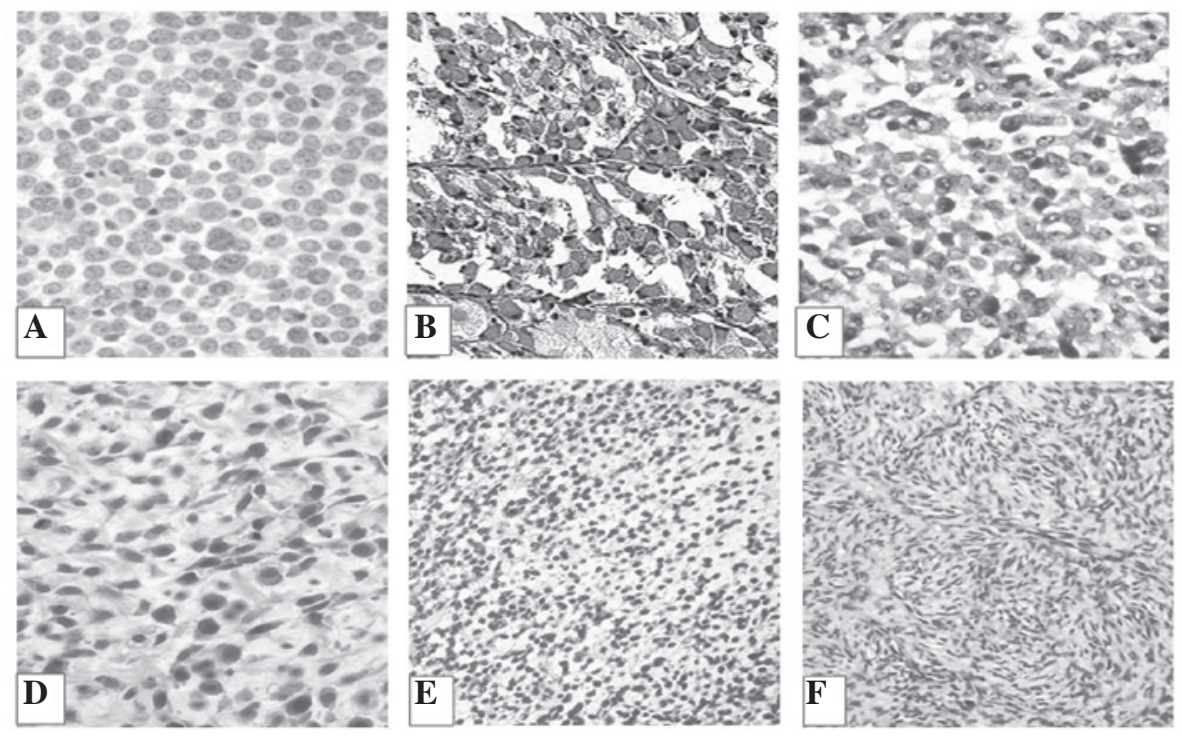

Figure 2. Histomorphological results of the tumors (hematoxylin and eosin stain). (A-F) Micrographs of (A) Ewing's sarcoma (malignant small round cell tumor), (B) alveolar RMS, (C) embryonal RMS, (D) osteosarcoma, (E) synovial sarcoma and (F) fibrosarcoma. RMS, rhabdomyosarcoma. Magnification, x40.

therapy. The aim of this study was to identify the best possible procedure for chest wall neoplasms with less potential risk and an increased survival rate.

\section{Materials and methods}

Study design and ethical approval. The present study was carried out as a retrospective analysis of chest wall tumors diagnosed in the Department of Cardiopulmonary Surgery of Xiangya Hospital (Changsha, China) between February 1, 2007 and July 31, 2013. This study was approved by Ethics Committee of Xiangya Hospital and further necessary permission from the patients for the conduction of the study was also obtained.

Patients. A total of 50 patients were observed in this study. The patients were analyzed on the basis of their gender, age (children, 4-8 years; young adult, 17-29 years; and adult, 41-50 years), disease symptoms, tumor location and histology/type of tumor. Of all the patients, 37 (74\%) were males and $13(26 \%)$ were females (Table I). Only primary tumors of the ribs, scapula, clavicle, sternum and their associated soft tissues were included. All the patients were diagnosed according to the updated clinical diagnostic criteria (2) and were further followed-up postoperatively over a period ranging from 14 months to six years.

Initial diagnostic techniques, such as CT and MRI, were carried out on all selected patients. Chest wall CT images were reviewed by a certified CT radiologist from the Department of Cardiopulmonary Surgery (Xiangya Hospital). To further extend the limits of the diagnosis, a four-dimensional scan was carried out to confirm the different types of chest wall tumors among the patients. Based on the symptoms and physical observations of the chest, the patients were grouped into five categories, as shown in Fig. 1 and Table I. The micrographs of the tumor types are shown in Fig. 2. The CT/MRI results revealed that, out of the 50 patients, 14 (28\%) showed the common symptoms of malignant small round 


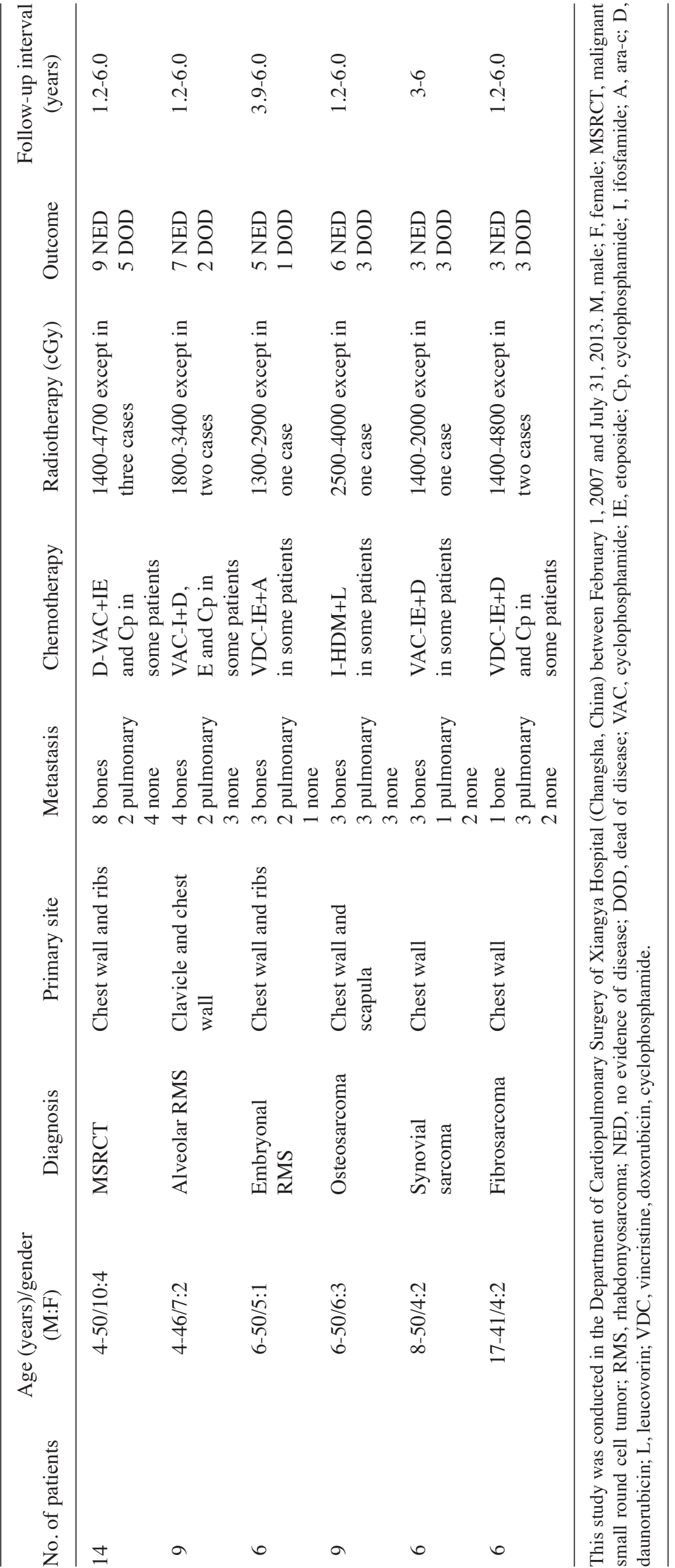


cell tumors (MSRCTs)/Ewing's sarcoma, and these patients were tested with MIC2 marker (10) for confirmation of the MSRCT diagnosis. Fifteen (30\%) patients were diagnosed with RMS, exhibiting symptoms such as swelling in the body and a persistent lump in the chest wall, and these diagnoses were further confirmed by screening tests with myogenin marker (11). Six patients were suffering from synovial sarcoma, which was confirmed by transduction-like enhancer gene 1 marker testing (12). Nine out of the 50 patients were sent for physical examination as they exhibited visible marks of swelling and redness of various body parts. A diagnosis of osteosarcoma was confirmed by performing blood tests with $\mathrm{C}-\mathrm{X}-\mathrm{C}$ chemokine receptor type 4 markers (13). The remaining six patients were reported to be suffering from fibrosarcoma (Fig. 1 and Table I).

Clinical evaluation and patient diagnosis. A biopsy was performed for confirmation according to previous studies $(14,15)$. A total of 10 out of 50 (20\%) patients were referred for biopsy, out of which seven underwent excisional biopsy and the three underwent incisional/core biopsy. The detailed treatment of the patients suffering from the reported chest wall malignancies is summarized in Table I.

Surgical methods. The surgical approaches were selected based on the extent of the tumor. Forty patients were exposed to tumor surgical resection through a posterior approach based on mild metastasis. Eleven patients with MSRCTs, 12 with rhabdomyosarcoma, eight with osteosarcoma, five with synovial sarcoma and four patients with fibrosarcoma were subjected to resection (Table II).

Radiation therapy. Radiotherapy is usually applied to the cancerous tumor. A total of 15 out of 40 patients were treated with image-guided radiation therapy (IGRT) and 20 with brachytherapy. The remaining five patients underwent intensity-modulated radiation therapy (IMRT).

\section{Results}

Patient data. A total of 50 patients varying in age and gender were selected for this study of the different types of sarcoma of the chest wall. The majority of the patients showed a positive response to the various diagnostic techniques used for their recovery; however, in certain cases the severity of the disease resulted in the mortality of the patient. In general, the survival rate for each studied chest wall tumor was slightly higher than the corresponding morbidity rate (Fig. 3). Techniques such as chemotherapy and radiotherapy have been shown to produce effective results in numerous neoplasms, including those of the chest wall (6), but in certain cases in the present study they were unable to increase the survival rates of the suffering patients to a satisfactory level. The survival rate of patients suffering from osteosarcoma (78\%) was higher than that of patients with RMS (73\%), followed by that of patients with MSRCT (64\%). In the cases of synovial sarcoma and fibrosarcoma, the survival and mortality rates of the suffering patients were equal, as shown in Fig. 3. Certain patients from each of the five groups succumbed due to disease progression, despite being subjected to intensive regimes of chemotherapy and/or
Table II. Representative number of patients subjected to various types of surgical procedures.

\begin{tabular}{lc}
\hline Surgical procedure & Number of patients \\
\hline Chest wall resection & 24 \\
En bloc resection & 11 \\
Biopsy & 10 \\
Distal clavicle resection & 3 \\
Scapular resection & 2 \\
\hline
\end{tabular}

Patients were assigned a surgical procedure based on the diagnosis, treatment, severity of disease, recovery percentage and follow-up over a certain period of time.

radiotherapy over a period of one to six years. These patients included five suffering from MSRCT, four from RMS, two from osteosarcoma and three each from synovial sarcoma and fibrosarcoma (Table I).

Complications. Several patients experienced complications following chemotherapy (30\%). Furthermore, infections occurred in eight out of 14 patients with MSRCT. Additional surgery was therefore performed for these eight patients. Nine out of the 15 (60\%) patients with RMS experienced side effects, which included nausea, hair loss and the development of low blood cell counts. The survival rates with IGRT, IMRT and brachytherapy were found to be $\sim 5 \%$ with a follow-up of six years.

\section{Discussion}

Chest wall tumors have long represented a clinical challenge for surgeons. Incorrect diagnosis, incomplete resection and unsuccessful reconstruction of large thoracic wall defects have resulted in high rates of perioperative morbidity and mortality. The analysis of the prognosis of a large series of patients following resection has indicated that surgical treatment may be the best option for primary tumors and for selected secondary tumors of the chest wall (7). Furthermore, surgery has been demonstrated to be the most efficacious curative option for patients with chest wall tumors (16). Unlike metastatic cases, the overall five-year survival rates of patients with localized disease have been enhanced to $\sim 80 \%$ with the combined use of surgery, radiation therapy and chemotherapy (17).

The blue cell tumor Ewing's sarcoma, one of a group of cancers collectively known as the Ewing's sarcoma family of tumors (ESFT), is the third most common malignant chest wall tumor overall and is not uncommon in the pediatric and young adult populations. The ESFT constitutes an aggressive tumor family with high recurrence rates and a high likelihood of metastases $(18,19)$. RMS, on the other hand, is a relatively rare form of cancer but is the most common soft tissue sarcoma in children. The tumor usually manifests as an expanding mass and the treatment constitutes wide resection followed by radiotherapy and chemotherapy, resulting in a five-year survival rate of $\sim 70 \%$. Ewing's sarcoma and RMS arise and can 


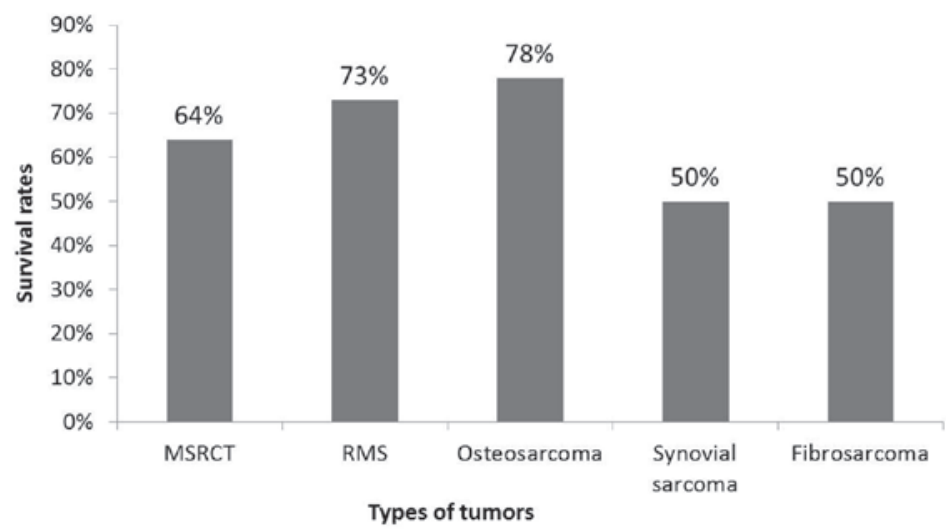

Figure 3. Survival rates of patients based on tumor types. RMS, rhabdomyosarcoma; MSRCT, malignant small round cell tumor.

extend into soft tissue in up to $6.5 \%$ (20) and $5 \%$ of pediatric patients (21). In the present study, the most frequent tumor sites were found to be a single rib (48\%), followed by the chest wall (34\%), clavicle (12\%) and scapula (6\%). These findings were in contrast to previous studies, in which the most common site of primary tumor occurrence was reported to be the rib $(22,23)$. Treatment regimens for MSRCTs and RMS act to control the local disease and distant spread. Shimotake et al (24) reported that the local control of MSRCTs could be achieved with radiotherapy, depending on the location, size and volume of the primary tumor, whereas Paulino et al (18) demonstrated that doses of 5,000-6,000 cGy exhibited $80-85 \%$ effectiveness in patients. The majority of the selected patients in the present study were administered different doses of radiotherapy, ranging from 1,300 to $4,800 \mathrm{cGy}$, and the overall survival rates were $\sim 65 \%$ (Fig. 3 and Table I).

A previous study on treatment with vincristine, doxorubicin and cyclophosphamide, along with etoposide and ifosfamide, showed increased survival rates (14). In the present study, such drugs were administered to the selected patients in varying concentrations as per the chemotherapeutic requirement (Table I). The majority of the patients showed a positive response to the chemotherapeutic doses, with the exception of a few severe cases, which were later referred for alternative treatments. The current results were consistent with those published by Casey et al (25), which concluded that a course regimen consisting of the short-term, high-dose administration of multiple selective drugs was optimal for primitive neuroectodermal tumors and Ewing's sarcoma in children and young adults.

Fibrosarcoma is a tumor of mesenchymal cell origin that accounts for $<10 \%$ of soft tissue tumors (26). It occurs more frequently in males, typically in the lower extremities. The treatment for fibrosarcoma involves excision, usually combined with radiation therapy (27). The synovial sarcoma constitutes $\sim 10 \%$ of soft tissue sarcomas. Treatment for this type of sarcoma consists of radical or wide excision of the tumor with adjuvant therapy (chemotherapy and/or radiation therapy) (28). A previous report on fibrosarcoma revealed unsatisfactory results with chemotherapy, while the complete removal of the tumor resulted in survival without local reoccurrence (29). Unni et al (30) studied the significance of fibrosarcoma with a low survival rate of $30 \%$. In the present study, the patients with fibrosarcoma from the selected population of 50 diseased individuals were subjected to a combination of chemotherapy and radiotherapy, and exhibited a 50\% survival rate with a follow-up of approximately six years. The same results were obtained for patients with synovial sarcoma, where the survival rate was $50 \%$ with a follow-up period of three to six years (Table I). By contrast, osteosarcoma, a malignant mesenchymal neoplasm that rarely occurs in the thorax, was treated by a wide resection of the tumor, including the entire bone, rib, sternum and adjacent soft tissue.

The present study has shown a favorable survival rate in patients with chest wall tumors. Regarding radiotherapy, it is difficult to make any definite recommendations based on the present data due to the small number of patients; however, the local control of the disease is fundamental in the treatment process, as failure to achieve this control can result in the reappearance of the disease and the possible development of distant metastases. In the present study, the tumors and all involved structures were surgically removed through a wide excision, and chemotherapy was subsequently administered in a regimen similar to that used prior to surgery. Following chemotherapy, adjuvant radiotherapy was performed to remove the remaining tumor and destroy microscopic lymphatic and hematogenous deposits of cancer spread. In pre-operative planning, the most successful method of characterizing the type and extent of the tumor is through CT or MRI techniques. On the basis of our experience, it can be concluded that planning the most appropriate surgical procedure and determining pre-operative staging using CT is useful technique and can facilitate the detection of abnormalities. Chest wall tumors are a motivating analytic and therapeutic challenge for reconstructive surgeons, and histopathology reports and radiographic images should be evaluated prior to surgery. For numerous patients, a multidisciplinary approach may be most effective with regard to survival and long-term function. For patients with malignant and metastatic tumors of the chest wall, optimal outcomes can be achieved with complete surgical resection and appropriate reconstruction. Achieving local control of the disease can be challenging. The present study on a small group of patients provides a foundation for further studies on tumors of chest wall, which may, in turn, decrease cancer-based mortality rates. 


\section{Acknowledgements}

The authors would like to thank the General Hospital of Chengdu Military Region of PLA (Chengdu, China) for providing assistance with the histomorphological studies.

\section{References}

1. D'ddario G, Früh M, Reck M, Baumann P, Klepetko W and Felip E: Metastatic non-small-cell lung cancer: ESMO Clinical Practice Guidelines for diagnosis, treatment and follow-up. Ann Oncol 21: v116-v119, 2010.

2. Ma X, Lin C and Zhen W: Cancer care in China: A general review. Biomed Imaging Interv J 4: e39, 2008.

3. He J, Gu D, Wu X, et al: Major causes of death among men and women in China. N Engl J Med 353: 1124-1134, 2005.

4. David EA and Marshall MB: Review of chest wall tumors: a diagnostic, therapeutic, and reconstructive challenge. Semin Plast Surg 25: 16-24, 2011.

5. Tateishi U, Gladish GW, Kusumoto M, et al: Chest wall tumors: radiologic findings and pathologic correlation: part 2. Malignant tumors. Radiographics 23: 1491-1508, 2003.

6. Smith SE and Keshavjee S: Primary chest wall tumors. Thorac Surg Clin 20: 495-507, 2010.

7. La Quaglia MP: Chest wall tumors in childhood and adolescence. Semin Pediatr Surg 17: 173-180, 2008.

8. Shah AA and D'Amico TA: Primary chest wall tumors. J Am Coll Surg 210: 360-366, 2010.

9. Kim JY and Hofstetter WL: Tumors of the mediastinum and chest wall. Surg Clin North Am 90: 1019-1040, 2010.

10. Ambros IM, Ambros PF, Strehl S, Kovar H, Gadner H and Salzer-Kuntschik M: MIC2 is a specific marker for Ewing's sarcoma and peripheral primitive neuroectodermal tumors Evidence for a common histogenesis of Ewing's sarcoma and peripheral primitive neuroectodermal tumors from MIC2 expression and specific chromosome aberration. Cancer 67: 1886-1893, 1991

11. Kumar S, Perlman E, Harris CA, Raffeld M and Tsokos M: Myogenin is a specific marker for rhabdomyosarcoma: an immunohistochemical study in paraffin-embedded tissues. Mod Pathol 13: 988-993, 2000.

12. Terry J, Saito T, Subramanian S, et al: TLE1 as a diagnostic immunohistochemical marker for synovial sarcoma emerging from gene expression profiling studies. Am J Surg Pathol 31: 240-246, 2007.

13. Huang CY, Lee CY, Chen MY, et al: Stromal cell-derived factor-1/CXCR4 enhanced motility of human osteosarcoma cells involves MEK1/2, ERK and NF-kappaB-dependent pathways. J Cell Physiol 221: 204-212, 2009.
14. Hsu PK, Lee HC, Hsieh CC, et al: Management of primary chest wall tumors: 14 years' clinical experience. J Chin Med Assoc 69: 377-382, 2006.

15. Soyer T, Karnak I, Ciftci AO, Senocak ME, Tanyel FC and Büyükpamukçu N: The results of surgical treatment of chest wall tumors in childhood. Pediatr Surg Int 22: 135-139, 2006.

16. Tukiainen E: Chest wall reconstruction after oncological resections. Scand J Surg 102: 9-13, 2013.

17. Punyko JA, Mertens AC, Baker KS, Ness KK, Robison LL and Gurney JG: Long-term survival probabilities for childhood rhabdomyosarcoma. Cancer 103: 1475-1483, 2005.

18. Paulino AC, Nguyen TX and Mai WY: An analysis of primary site control and late effects according to local control modality in non-metastatic Ewing sarcoma. Pediatr Blood Cancer 48: 423-429, 2007.

19. van Rossen ME, Verduijn P and Mureau M: Survival of pedicled pectoralis major flap after secondary myectomy of muscle pedicle including transection of thoracoacromial vessels: does the flap remain dependent on its dominant pedicle? J Plast Reconstr Aesthet Surg 64: 323-328, 2011.

20. Chattopadhyay A, Nagendhar Y and Kumar V: Osteosarcoma of the rib. Indian J Pediatr 71: 543-544, 2004.

21. Dang NC, Siegel SE and Phillips JD: Malignant chest wall tumors in children and young adults. J Pediatr Surg 34: 1773-1778, 1999.

22. Burt M, Karpeh M, Ukoha O, et al: Medical tumors of the chest wall. Solitary plasmacytoma and Ewing's sarcoma. J Thorac Cardiovasc Surg 105: 89-96, 1993

23. Sabanathan S, Salama FD, Morgan WE and Harvey JA: Primary chest wall tumors. Ann Thorac Surg 39: 4-15, 1985.

24. Shimotake T, Fumino S, Aoi S, Tsuda T and Iwai N: Respiratory insufficiency in a newborn with mesenchymal hamartoma of the chest wall occupying the thoracic cavity. J Pediatr Surg 40: E13-E16, 2005.

25. Casey DA, Wexler LH, Merchant MS, et al: Irinotecan and temozolomide for Ewing sarcoma: The Memorial Sloan-Kettering experience. Pediatr Blood Cancer 53: 1029-1034, 2009.

26. Radaelli S, Stacchiotti S, Casali PG and Gronchi A: Emerging therapies for adult soft tissue sarcoma. Expert Rev Anticancer Ther 14: 689-704, 2014.

27. Folpe AL: Fibrosarcoma: a review and update. Histopathology 64: 12-25, 2014.

28. Thway K and Fisher C: Synovial sarcoma: defining features and diagnostic evolution. Ann Diagn Pathol 18: 369-380, 2014.

29. Papagelopoulos PJ, Galanis EC, Trantafyllidis P, Boscainos PJ Sim FH and Unni KK: Clinicopathologic features, diagnosis, and treatment of fibrosarcoma of bone. Am J Orthop (Belle Mead NJ) 31: 253-257, 2002.

30. Unni KK and Inwards CY (eds): Dahlin's Bone Tumors: General Aspects and Data on 10,165 Cases. Lippincott Williams \& Wilkins, Philadelphia, PA, 2010. 